\title{
An Improvement in a Local Observer Design for Optimal State Feedback Control: The Case Study of HIV/AIDS Diffusion
}

\author{
Paolo Di Giamberardino and Daniela Iacoviello \\ Dept. Computer, Control and Management Engineering Antonio Ruberti, Sapienza University of Rome, via Ariosto 25, \\ 00185 Rome, Italy
}

Keywords: $\quad$ Nonlinear Systems, Linear Observer, Optimal Control, LQR, Epidemic Spread.

\begin{abstract}
The paper addresses the problem of an observer design for a nonlinear system for which a preliminary linear state feedback is designed but the full state is not measurable. Since a linear control assures the fulfilment of local approximated conditions, usually a linear observer is designed in these cases to estimate the state with estimation error locally convergent to zero. The case in which the control contains an external reference, like in regulations problems, is studied, showing that the solution obtained working with the linear approximation to get local solutions produces non consistent results in terms of local regions of convergence for the system and for the observer. A solution to this problem is provided, proposing a different choice for the observer design which allows to obtain all conditions locally satisfied on the same local region in the neighbourhood of a new equilibrium point. The case study of an epidemic spread control is used to show the effectiveness of the procedure. The linear control with regulation term is present in this case because the problem is reconducted to a Linear Quadratic Regulation problem. Simulation results show the differences between the two approaches and the effectiveness of the proposed one.
\end{abstract}

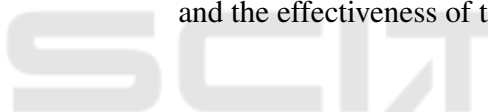

\section{INTRODUCTION}

The problem of the state measure for dynamical systems is an important aspect in the control theory for all the applications in which the control laws require the knowledge of a non fully measurable state. The history of solutions to this problem begins with the case of linear dynamics (Luenberger, 1964) and, less than ten years later, it is enriched with the first results for nonlinear ones.

Afterwards, several solutions have been presented in literature for the design of state observers. Clearly, the most large number of contributions refers to the case of nonlinear dynamics, for which nonlinear solutions have been proposed. One direction of the research activity is represented by nonlinear solutions which mainly follows the idea initially proposed in (Luenberger, 1964) for linear systems: an observer can be designed starting from a copy of the dynamics whom corrective terms are added to, aiming at the stabilization of the linear approximation of the observer and of the full interconnected system. Examples are (Andrieu and Praly, 2006), (Zeitz, 1987) and (Sundarapandian, 2006), where autonomous dynamics are considered. The importance of the stabilization of the linear part of the whole dynamics as an initially local solution is usually put in evidence separating explicitly the linear component of the system from the remaining nonlinear terms to better highlight the local behaviours, (Kazantzis and Kravaris, 1997). A further example of a solution based on the possibility of linearising the error dynamics is represented by (Respondek et al., 2004).

The explicit presence of the input in the nonlinear dynamics usually complicates the approaches, since suitable bounding conditions must be given, or different solutions (Sundarapandian, 2002) must be adopted, modelling the input as generated by an exosystem with known structure.

The list of references could be very long, till nowadays with, for example, (Sassano and Astolfi, 2019), where an approximated linearising feedback for the system dynamics is introduced.

In the larger part of their use, observers are part of feedback control schemes providing a state estimate for state feedback laws. Then, the control design and the observer determinations are two problems that must be solved at the same time. They can be solved separately in the linear case, where the Separation Principle allows to prove that the addition of an ob- 
server does not change the dynamical characteristics of the controlled system. In the nonlinear case, the Separation Principle can be also invoked once local liner approximations are considered, bringing back the problem to a linear one but restricting to local solutions.

The problem addressed in this paper refers to the cases in which the nominal state feedback, designed for satisfying prefixed requirements, changes the equilibrium point of the controlled system. In this case, the concept of local solution is no more valid since there exist at least two different reference points: the equilibrium for the initial given dynamics and the new one, the working point, equilibrium for the controlled system.

The approach here followed aims at defining a unique equilibrium point so that local conditions on both the control and on the observer can be satisfied in a neighbourhood of such a point.

Only local conditions are considered, strictly dependent on the regions in which they are defined and satisfied. Extensions to nonlinear observers can be performed adopting the same procedure known for many nonlinear approaches which start with the fulfilment of local specifications.

The proposed procedure is applied to a case study, represented by the control of an epidemic spread of a virus, the one responsible of HIV/AIDS infections.

The choice is motivated by the fact that the classical procedure, based on the linearisation of the nonlinear dynamics and the design of both the controller and the observer, satisfying local requirements, performed on the basis of the linearised system, has been proposed in (Di Giamberardino and Iacoviello, 2018) and can be assumed as a reference behaviour to compare the solutions here proposed.

A further motivation is that, despite the mathematical models of different epidemic spreads have peculiar characteristics for each different epidemic addressed, the control strategy usually adopted in literature makes reference to optimal solutions, like (Jun, 2006; Bakare et al., 2014; Di Giamberardino and Iacoviello, 2017) for the SIR (Susceptible-InfectiousRemoved subjects), (Lin et al., 2010; Gupar and Quanyan, 2013; Iacoviello and Stasio, 2013) for the influenza, (Supriatna et al., 2016) for the dengue, and so on.

The reason of such a choice is that in these cases the control actions, represented for example by prevention, vaccination, medication, quarantine, are all subject to a possible limitation and the distribution of the resources among the different controls can be handled by means of suitable cost functions.

To better understand the mathematical model of the particular case study adopted, some concepts are shortly reported. The HIV/AIDS virus attacks the cells of the immune system, damaging them so that the immune system is inhibited and the individual is less protected against other infections. Virus transmission is facilitated by contacts with infected body fluids. The AIDS represents the most advanced stage of the HIV infection; it can be reached in 10-15 years from the initial HIV infection. Up to now, the control actions are the prevention and the medication after a positive diagnosis.

The mathematical modelling of the HIV/AIDS diffusion among populations is focused on the dynamic of interactions between individuals (Wodarz and Nowak, 1999; Pinto and Rocha, 2012; Di Giamberardino et al., 2017; Di Giamberardino et al., 2018).

In classical HIV/AIDS spread models (Naresh et al., 2009; Wodarz and Nowak, 1999; Chang and Astolfi, 2009), four main classes are introduced: the Susceptible subjects (S), that are the healthy people that may contract the virus, the Infectious one (I) that are individuals not aware of their condition, the preAIDS patients (P), the AIDS patients (A). The control actions introduced are mainly focused on the prevention, as for example in (Rutherford et al., 2016), where the attention is devoted to risky subjects, drug users and sex workers.

In this paper, the model proposed in (Di Giamberardino et al., 2017; Di Giamberardino et al., 2018) and used in (Di Giamberardino and Iacoviello, 2018) is adopted, for comparative purpose. The main difference between this and the classical models is that the susceptible individuals, $\mathrm{S}$, are here divided into two categories, distinguishing those who adopts wise behaviours from the ones that do not take into account the dangerousness of the disease. Moreover, the model follows the suggestions of the World Health Organization (WHO) for the control input: i. actions aiming at reducing the possibility of new infections, through informative campaign, for example; ii. facilitations of a fast diagnosis for unaware infectious patients, thus reducing the percentage of subjects responsible of the virus spread; iii. medication support to the aware infectious subjects.

Following (Di Giamberardino and Iacoviello, 2018), the control problem is formulated in the framework of optimal control theory, introducing a cost function which weights the number of unaware infectious individuals $I(t)$ and the controls introducing a quadratic cost index. This particular form suggested to find a solution in the context of a LQR problem, passing through the linearisation of the dynamics in a neighbourhood of one of its equilibrium points. The 
solution is a linear state feedback with a constant reference contribution arising from the regulation problem.

Unfortunately, for its implementation, the control scheme needs a state observer, since only the number of the patients with HIV (P) and AIDS (A) are available. As already discussed, the observer design can be performed working on the model linearisation already adopted for the LQR problem solution, as in (Di Giamberardino and Iacoviello, 2018).

This approach and the improvement introduced to overcome some consequent consistency problems are discussed in the paper. More in details, in Section 2 the inconsistencies of the straightforward approach which works on the same linearised dynamics are put in evidence, while the improvements for overcoming such a problem are presented and discussed in Section 3. The procedure is then applied to the case study in Section 4, comparing it with the simpler classical one. Some results of numerical simulations are reported in Section 5 to validate the proposed solution. Some concluding remarks in Section 6 end the paper.

\section{PROBLEM DEFINITION}

Given the nonlinear dynamics

$$
\begin{aligned}
\dot{x} & =f(x)+g(x) u \\
y & =h(x)
\end{aligned}
$$

with $x \in \mathfrak{R}^{n}, u \in \mathfrak{R}^{m}, y \in \mathfrak{R}^{p}$, and the equilibrium point $x_{e}\left(f\left(x_{e}\right)=0, g\left(x_{e}\right) \neq 0, h\left(x_{e}\right)=0\right)$, the problem here studied refers to the case in which the design of an observer is required for the implementation of a state feedback control. As discussed in the Introduction, the majority of the approaches for the determination of an observer for a nonlinear system aims at obtaining local asymptotic convergence, in a neighbourhood of one equilibrium point, with the addition of further conditions on the strictly nonlinear part of the dynamics, and on the input, when necessary, to extend the region of convergence or to make the result global.

So, if one restricts the attention to linear feedback control law, satisfactory once only local conditions on the control are required, then also for the observer the design can be restricted to satisfy local conditions only, so simplifying the computation and, sometimes, its implementation.

Under these hypothesis, the problem can be approached computing firstly the linear approximation of (1)-(2), getting

$$
\begin{aligned}
\dot{\tilde{x}} & =A \tilde{x}+B u \\
\tilde{y} & =C \tilde{x}
\end{aligned}
$$

where, as usual, $A=\left.\frac{\partial f}{\partial x}\right|_{x=x_{e}}, B=g\left(x_{e}\right), C=\left.\frac{\partial h}{\partial x}\right|_{x=x_{e}}$, $\tilde{x}=x-x_{e}$ and $\tilde{y}=y-C x_{e}$. Based on (3), the linear feedback control and the linear observer can be easily computed working in the linear context.

In the present work, the case in which the linear feedback control computed assumes the form

$$
u=K \tilde{x}+r
$$

is considered. The linear term $K \tilde{x}$ satisfies the local stability of the controlled system in a neighbourhood of the equilibrium point. The additional presence of a forcing constant term $r$ in (4) is considered, which usually appears when dealing with a regulation problem, where the external input plays the role of a reference value. In Section 4 a real case is introduced to show an example of synthesis in which the regulation term naturally appears.

In this case of whole linear approximation and local solutions, the closed loop dynamics under state measurement would become

$$
\dot{\tilde{x}}=(A+B K) \tilde{x}+B r
$$

The linear observer may assume the classical Luenberger form (Luenberger, 1964)

$$
\dot{\tilde{z}}=(A-G C) \tilde{z}+B u+G \tilde{y}
$$

The dynamics of the error $e=\tilde{z}-\tilde{x}$ locally in a neighbourhood of $x_{e}$ is described by

$$
\begin{aligned}
\dot{e} & =(A-G C) \tilde{z}+B u+G \tilde{y}-(A+B K) \tilde{x}-B r= \\
& =(A-G C) e
\end{aligned}
$$

asymptotically convergent to zero once $\sigma(A-G C) \in$ $C^{-}$. Then, the asymptotic condition $\lim _{t \rightarrow \infty}\|\tilde{z}-\tilde{x}\|=0$ holds and it can be rewritten as

$$
\lim _{t \rightarrow \infty}\|\tilde{z}-\tilde{x}\|=\lim _{t \rightarrow \infty}\left\|\tilde{z}+x_{e}-x\right\|=0
$$

showing that if $\tilde{z}$ is the estimate of $\tilde{x}$, then $z=\tilde{z}+x_{e}$ is the estimate of the original state $x$.

Remaining in the approximated context, the whole system obtained using the state reconstructed by the observer in the control law (4) is described by

$$
\begin{aligned}
\dot{\tilde{x}} & =A \tilde{x}+B K \tilde{z}+B r \\
\dot{\tilde{z}} & =(A-G C) \tilde{z}+B K \tilde{z}+B r+G C \tilde{x}
\end{aligned}
$$

and, replacing the observer dynamics with the one of the estimation error $e=\tilde{z}-\tilde{x}$, the full dynamics is given by

$$
\begin{aligned}
\dot{\tilde{x}} & =(A+B K) \tilde{x}+B K e+B r \\
\dot{e} & =(A-G C) e
\end{aligned}
$$

that is the proof of the Separation Principle. 
This procedure has been used in (Di Giamberardino and Iacoviello, 2018) for the optimal control of an epidemic spread. The example is recalled in next Section 4 and it is used to show the new result discussed later in the paper.

A weakness of the procedure described above can be put in evidence once the solution is applied to the original nonlinear model.

In order to analyse the effects of each contribution in the whole controlled system, the use of the nominal state feedback (4) is firstly introduced. The controlled dynamics can be written as

$$
\dot{x}=f(x)+g(x)\left(K\left(x-x_{e}\right)+r\right)=F_{c}(x, r)
$$

Computing the equilibrium points, denoted as $x_{e}^{c}$ to put in evidence its origin from the controlled dynamics, one has

$$
f\left(x_{e}^{c}\right)+g\left(x_{e}^{c}\right)\left(K\left(x_{e}^{c}-x_{e}\right)+r\right)=F_{c}\left(x_{e}^{c}, r\right)=0
$$

It is easy to verify that if $r=0, x_{e}^{c}=x_{e}$. Otherwise, the new equilibrium point $x_{e}^{c}$ is different from $x_{e}$.

This change implies that, at steady state, the system is in the equilibrium point $x_{e}^{c}$.

The introduction of an observer to estimate the state for a feedback implementation must preserve this asymptotic behaviour, as it happens in the linear case, and the equilibrium point must remain $x_{e}^{c}$.

The fulfilment of this condition can be verified analysing the whole system obtained introducing the estimated state given by the observer for the state feedback (13) applied to system (1).

On the basis of the relationships between the local state $\tilde{x}$ and its estimate $\tilde{z}$, as well as between the original state $x$ and its estimate $z$, the control law (4) can be expressed, in the original coordinates, as

$$
u=K z-K x_{e}+r
$$

and the observer dynamics (6) can assume the form

$$
\begin{aligned}
\dot{z} & =(A-G C)\left(z-x_{e}\right)+B u+G y-G C x_{e}= \\
& =(A-G C) z-A x_{e}+B u+G y
\end{aligned}
$$

The full interconnected dynamics is then described by

$$
\begin{aligned}
\dot{x} & =f(x)+g(x) K z-g(x) K x_{e}+g(x) r \\
\dot{z} & =(A-G C+B K) z-(A+B K) x_{e}+G C x+B r
\end{aligned}
$$

In order to check the effectiveness of the controlled system (15), as a first step the error dynamics like (7) can be computed to verify its convergence to zero.

$$
\begin{aligned}
\dot{e}= & (A-G C+B K-g(x) K) e \\
& +(A+B K-g(x) K) x-f(x) \\
& -(A+B K-g(x) K) x_{e}+(B-g(x)) r
\end{aligned}
$$

Approximating (16) in a neighbourhood of $x=x_{e}$, one gets

$$
\dot{e}=(A-G C) e
$$

the same as in (10) as expected. That is, while the system converges to $x_{e}$, the estimation error goes to zero.

The problem is that, for the full dynamics (15), $x_{e}$ is not an equilibrium point, as it is easy to verify just substituting $x=x_{e}$ in

$$
\begin{aligned}
f(x)+g(x) K z-g(x) K x_{e}+g(x) r & =0 \\
(A-G C+B K) z-(A+B K) x_{e}+G C x+B r & =0
\end{aligned}
$$

as well as $z=x_{e}$ since the estimation error goes to zero. The condition

$$
B r=0
$$

is obtained, clearly impossible. On the other hand, not even $x=x_{e}^{c}$, and then $z=x_{e}^{c}$, are equilibrium conditions for the two subsystems because, by substitution in (18), the expressions

$$
\begin{aligned}
-g\left(x_{e}^{c}\right) K x_{e} & =0 \\
(A+B K)\left(x_{e}^{c}-x_{e}\right)+B r & =0
\end{aligned}
$$

are obtained, again impossible.

It is possible to conclude that this approach cannot work properly because $i$. the insertion of the observer dynamics interferes with the characteristics of the controlled system, changing the equilibrium point; $i$. the observer does not work as expected, since the manifold in which the local convergence is assures does not coincide with a neighbourhood of the new equilibrium point.

The goal of the present paper is to introduce an improvement in the procedure recalled above, remaining in the locally linearised approximated context but avoiding the undesired effects $i$. and $i i$. described above.

\section{THE PROPOSED DESIGN PROCEDURE}

The idea followed for the solution here proposed is based on the possibility of designing a state observer in such a way that the equilibrium point of the controlled system is the same both when the state is supposed to be measured and when its estimate provided by the observer is used.

Starting from the system (1)-(2), suppose it has been defined a linear state feedback control with a 
regulation term of the form (4), expressed in the original coordinates,

$$
u=K x+r
$$

The controlled dynamics is described by

$$
\dot{x}=f(x)+g(x) K x+g(x) r=F(x, r)
$$

with output (2). Using the same notation previously adopted, be $x_{e}^{c}$ the equilibrium point for the controlled system (22), $F\left(x_{e}^{c}, r\right)=0$.

The design technique is again based on a linear observer and on local convergence of the estimation error, but preserving the convergence of the system to $x_{e}^{c}$.

To this aim, the linear approximation of (22) in a neighbourhood of $x_{e}^{c}$ is computed as

$$
\dot{\bar{x}}=A_{c} \bar{x}
$$

where $\bar{A}=\left.\frac{\partial F(x, r)}{\partial x}\right|_{x=x_{e}^{c}}$ and $\bar{x}=x-x_{e}^{c}$. Now, a linear observer is designed on the basis of the closed loop system, i.e. an observer for the state of (22). The structure is the same as in (6), so that it has the form

$$
\dot{\bar{z}}=A_{c} \bar{z}+G(\bar{y}-C \bar{z})=\left(A_{c}-G C\right) \bar{z}+G \bar{y}
$$

where $\bar{y}=y-C x_{e}^{c}$ is defined as in the previous case for a different equilibrium point. Conditions under which the estimation error converges asymptotically to zero for the so defined problem are trivial, being $\sigma\left(A_{c}-G C\right) \in C^{-}$.

The so obtained observer is used in the full closed loop system to provide a state estimation for the state feedback (21). Clearly, since $\bar{z}$ is the estimation of $\bar{x}$, that is $\lim _{t \rightarrow \infty}\|\bar{x}-\bar{z}\|=0, z=\bar{z}+x_{e}^{c}$ is the estimation of $x$; in fact $\lim _{t \rightarrow \infty}\|\bar{x}-\bar{z}\|=\lim _{t \rightarrow \infty}\left\|\left(x-x_{e}^{c}\right)-\left(z-x_{e}^{c}\right)\right\|=$ $\lim _{t \rightarrow \infty}\|x-z\|=0$

In order to study the effect of such a control scheme, the full closed loop dynamics has to be written. One has

$$
\begin{aligned}
\dot{x} & =f(x)+g(x) K z+g(x) r \\
& =F(x, r)+g(x) K(z-x) \\
\dot{z} & =\left(A_{c}-G C\right) z-A_{c} x_{e}^{c}+G C x
\end{aligned}
$$

If the dynamics of the error $e=z-x$ is computed, the expression

$$
\begin{aligned}
\dot{e}= & \left(A_{c}-G C\right)(e+x)-A_{c} x_{e}^{c}+G C x-F(x, r) \\
& -g(x) K e= \\
= & \left(A_{c}-G C-g(x) K\right) e+A_{c}\left(x-x_{e}^{c}\right)-F(x, r)
\end{aligned}
$$

is obtained. Its approximation in a neighbourhood of $x=x_{e}^{c}$ can be computed, setting

$$
F(x, r)=A_{c}\left(x-x_{e}^{c}\right)
$$

and

so yielding

$$
B_{c}=g\left(x_{e}^{c}\right)
$$

$$
\dot{e}=\left(A_{c}-G C-B_{c} K\right) e
$$

which converges, if the pair $\left(A_{c}-B_{c} K, C\right)$ is detectable, finding $G$ such that $\sigma\left(A_{c}-B_{c} K-G C\right) \in C^{-}$.

At the same time, once the equilibrium points of (25) are computed, it is easy to verify, by straightforward substitution, that $x=x_{e}^{c}$ and $z=x_{e}^{c}$ are one solution. In fact

$$
\begin{aligned}
F\left(x_{e}^{c}, r\right)+g\left(x_{e}^{c}\right) K\left(x_{e}^{c}-x_{e}^{c}\right) & =0 \\
\left(A_{c}-G C\right) x_{e}^{c}-\left(A_{c}-G C\right) x_{e}^{c} & =0
\end{aligned}
$$

The fulfilment of the Separation Principle can be verified, rewriting (25) in the new coordinates $(x, e)$ :

$$
\begin{aligned}
\dot{x} & =F(x, r)+g(x) K e \\
\dot{e} & =\left(A_{c}-G C-g(x) K\right) e+A_{c}\left(x-x_{e}^{c}\right)-F(x, r)
\end{aligned}
$$

which, in a neighbourhood of $x=x_{e}^{c}$ can be approximated as

$$
\begin{aligned}
\dot{x} & =A_{c} x+B_{c} K e-A_{c} x_{e}^{c} \\
\dot{e} & =\left(A_{c}-G C-B_{c} K\right) e
\end{aligned}
$$

whose dynamical matrix is

$$
A_{T O T}=\left(\begin{array}{cc}
A_{c} & B_{c} K \\
0 & A_{c}-G C-B_{c} K
\end{array}\right)
$$

proving that the Separation Principle still holds.

A comparison between the two approaches, the first with the equilibrium point change and an error on the state estimation, and the second, for which the equilibrium is left unchanged and the Separation Principle holds, is reported in next Section 4. In particular, the first approach is recalled referring to (Di Giamberardino and Iacoviello, 2018) and it is used to compare the results obtained with the here proposed design procedure.

\section{THE CASE STUDY}

\subsection{A Short Recall of the Mathematical Model}

In this paper, the model of the HIV/AIDS diffusion presented in (Di Giamberardino et al., 2017; Di Giamberardino et al., 2018) is adopted and is here briefly recalled. Main characteristics of this epidemic are the 
possibility of spread limitation by means of a healthy behaviour of individuals, while vaccination is still not available; moreover, there can be a quite long period during which infected persons are infective but unconscious of their status, since the symptoms appear later.

The first point motivates the introduction of a control based on suitable informative and educational campaign, to reduce unhealthy relationships, instead of a more classical vaccination action, and of a control aiming to discover infected individuals as soon as possible, through a blood test campaign.

The state variables introduced in the model denote the healthy people $S_{1}$, not aware of dangerous behaviours and then can be infected, and $S_{2}$, the ones that, suitably informed, give great attention to the protection, and the three levels of infectious subjects: $I$, the infective but unaware of their status, $P$, the HIV positive patients, $A$, the AIDS diagnosed ones.

As previously discussed, the control actions are the information campaign, $u_{1}$ and the test campaign to discover the infection as soon as possible, $u_{2}$. A third action, $u_{3}$, the therapy which aims at reducing the transition from HIV to AIDS, is also considered, since mortality among $A$ is higher that in $P$.

Then, the mathematical model is

$$
\begin{aligned}
\dot{S}_{1}= & Z-d S_{1}-\beta \frac{S_{1} I}{N_{c}}+\gamma S_{2}-S_{1} u_{1} \\
\dot{S}_{2}= & -(\gamma+d) S_{2}+S_{1} u_{1} \\
\dot{I}= & \beta \frac{S_{1} I}{N_{c}}-(d+\delta) I-\psi \frac{I}{N_{c}} u_{2} \\
\dot{P}= & \varepsilon \delta I-(\alpha+d) P+\phi \psi \frac{I}{N_{c}} u_{2}+P u_{3} \\
\dot{A}= & (1-\varepsilon) \delta I+\alpha P-(\mu+d) A+ \\
& +(1-\phi) \psi \frac{I}{N_{c}} u_{2}-P u_{3}
\end{aligned}
$$

where $N_{c}=S_{1}+S_{2}+I$. In (33), $d$ denotes the rate of natural death; $Z$ denotes the flux of new subjects in the class $S_{1} ; \beta$ is related to the dangerous interactions between $S_{1}$ and $I$ categories; $\gamma$ is the rate of wise subjects that could change, incidentally, their status, increasing $S_{1}(t) ; \psi$ is related to the control action aiming at helping the individuals in class $I$ to discover their infectious condition, and therefore to flow to the $P$ or the $A$ class; $\phi$ is the percentage of test positive subjects with HIV $((1-\phi)$ the percentage with AIDS); $\delta$ is the rate of transition from $I$ to $P$ (percentage $\varepsilon$ ) or $A$ (percentage $(1-\varepsilon)$ ) without any external action; $\alpha$ is the rate of the natural transition from $P$ to $A ; \mu$ is the rate of death in class $A$ caused by the infection.

Dynamics (33) can also be expressed in the com- pact form

$$
\dot{X}=f(X)+g(X) U=F(X, U)
$$

once $X=\left(\begin{array}{llll}S_{1} & S_{2} I & P & A\end{array}\right)^{T}, U=\left(\begin{array}{lll}u_{1} & u_{2} & u_{3}\end{array}\right)^{T}$ and

$$
f(X)=\left(\begin{array}{c}
Z-d S_{1}-\frac{\beta S_{1} I}{N_{c}}+\gamma S_{2} \\
-(\gamma+d) S_{2} \\
\frac{\beta S_{1} I}{N_{c}}-(d+\delta) I \\
\varepsilon \delta I-(\alpha+d) P \\
(1-\varepsilon) \delta I+\alpha P-(\mu+d) A
\end{array}\right)
$$

$g(X)=\left(\begin{array}{lll}g_{1} & g_{2} & g_{3}\end{array}\right)=\left(\begin{array}{ccc}-S_{1} & 0 & 0 \\ S_{1} & 0 & 0 \\ 0 & -\psi \frac{I}{N_{c}} & 0 \\ 0 & \phi \psi \frac{I}{N_{c}} & P \\ 0 & (1-\phi) \psi \frac{I}{N_{c}} & -P\end{array}\right)$

are defined.

For the definition of possible output functions, it must be observed that the subject with a positive diagnosis, $P$ and $A$, can be easily measured, since reported by medical operators. On the other hand, individuals I cannot be known at all: they can be discovered only after blood test or symptoms rise, but in these case they are counted as $P$ or $A$. Consequently, also $S_{1}$ and $S_{2}$ can be known. The entire population is another measurable quantity.

Then, it seems reasonable to assume the measure of the total number of diagnosed individuals, $P(t)+$ $A(t)$, as the possible meaningful output, so giving

$$
y(t)=C X(t), \quad C=\left(\begin{array}{lllll}
0 & 0 & 0 & 1 & 1
\end{array}\right)
$$

\subsection{The Control Problem Formulation}

In this Section the control problem is shortly discussed showing one of the cases in which the control law is a linear state feedback with an additional constant reference term. An optimal control problem for the HIV/AIDS dynamics (33) has been formulated in (Di Giamberardino and Iacoviello, 2018) under the following assumptions: i. the most dangerousness aspect in the epidemic spread is represented by the individuals $I$, so that their minimization was the main goal; ii. once a diagnosis is given, there is no difference between $P$ and $A$ with respect to the decrement of $I$, so the therapy $u_{3}$ does not influence $I$ at all and it will be not considered, setting it to zero (any value would have the same effects on $I$ ). Then, the two-dimensional control vector $\hat{U}=\left(u_{1} u_{2}\right)^{T}$ is introduced, neglecting, consequently, the vector field $g_{3}(\cdot)$ in (36), introducing also the matrix $\hat{g}(X)=\left(\begin{array}{ll}g_{1} & g_{2}\end{array}\right)$. 
Under these positions, the cost function

$$
\begin{aligned}
J(X, \hat{U}) & =\frac{1}{2} \int_{t_{0}}^{\infty}\left(q I^{2}+r_{1} u_{1}^{2}+r_{2} u_{2}^{2}\right) d t= \\
& =\frac{1}{2} \int_{t_{0}}^{\infty}\left(X^{T} Q X+\hat{U}^{T} R \hat{U}\right) d t
\end{aligned}
$$

is defined, with $Q$ the five dimensional square matrix with all zero entries except $Q(3,3)=q$, and $R=\left(\begin{array}{cc}r_{1} & 0 \\ 0 & r_{2}\end{array}\right), r_{1}, r_{2}>0$. In other words, the goal of the proposed control action is the minimization of the number of infectious subjects $I$ making use of as less resources as possible.

The quadratic structure of (38) and the preference for a state feedback implementability of the control law drove the solution of such problem in (Di Giamberardino and Iacoviello, 2018) to a LQR form designed on the linearised approximation of (33) in the neighbourhood of one equilibrium point.

A study of the existence of equilibrium points and of their stability properties has been performed in (Di Giamberardino et al., 2017; Di Giamberardino et al., 2018), yielding to the two possible solutions

$$
X_{1}^{e}=\left(\begin{array}{c}
1 / d \\
0 \\
0 \\
0 \\
0
\end{array}\right) Z \quad X_{2}^{e}=\left(\begin{array}{c}
1 / H \\
0 \\
\frac{H-d}{H(d+\delta)} \\
\frac{\varepsilon \delta(H-d)}{H(\alpha+d)(d+\delta)} \\
\frac{\delta(H-d)[(1-\varepsilon) d+\alpha]}{H(\alpha+d)(d+\delta)(\mu+d)}
\end{array}\right) Z
$$

where $H=\beta-\delta$. The non negativeness of the elements in the vector state $X_{2}^{e}$ implies the conditions $H>0$ and $H \geq d$; therefore the equilibrium point $X_{2}^{e}$ is a feasible one if and only if $H \geq d$, being $X_{1}^{e}=X_{2}^{e}$ if $H=d$. A bifurcation analysis is reported in (Di Giamberardino et al., 2018).

Making use of the same values for the model parameters as in (Di Giamberardino and Iacoviello, 2018), condition $H>d$ holds, so that both the equilibrium points exist. Computing the two linearised dynamics in the neighbourhood of the two equilibrium points one gets

$$
\begin{aligned}
\dot{\tilde{X}} & =A_{i} \tilde{X}+\hat{B}_{i} \hat{U} \\
\tilde{y} & =C \tilde{X}
\end{aligned}
$$

with $A_{i}=\frac{\partial F}{\partial X}, \hat{B}_{i}=\frac{\partial F}{\partial \hat{U}}=\hat{g}$, all evaluated at $X=X_{i}^{e}$ and $\hat{U}=0, \tilde{y}=C \tilde{X}=y-C X_{i}^{e}$ and

$$
\tilde{X}=X-X_{i}^{e}
$$

$i=1,2$ depending on the choice.

Despite the procedure can be adopted making reference to both the equilibrium points, easy computations show that the linear dynamics which approximates the nonlinear one in the neighbourhood of $X_{1}^{e}$ is neither detectable nor controllable. So, in view of a control synthesis in the local linear domain, the linearisation in a neighbourhood of $X_{2}^{e}$ is chosen. Then, the linear dynamics is (40) with $i=2$.

\subsection{The Optimal Control Problem Solution}

The use of the new coordinates $\tilde{X}$, consequence of (41), implies a change of variables also in the cost function (38), where $\tilde{X}$ must appear instead of $X$. The new expression is

$$
\begin{aligned}
& J(X, \hat{U})=J\left(\tilde{X}+X_{2}^{e}, \hat{U}\right)=\tilde{J}(\tilde{X}, \hat{U})= \\
& =\frac{1}{2} \int_{t_{0}}^{\infty}\left(\left(\tilde{X}^{T}-\bar{r}\right) Q(\tilde{X}-\bar{r})+\hat{U}^{T} R \hat{U}\right) d t \\
& =\frac{1}{2} \int_{t_{0}}^{\infty}\left(q\left(\tilde{I}(t)+\frac{H-d}{H(d+\delta)}\right)^{2}+r_{1} u_{1}^{2}(t)+r_{2} u_{2}^{2}(t)\right) d t
\end{aligned}
$$

where $\bar{r}=\left(* * \bar{r}_{\tilde{I}} * *\right)^{T}$ denotes the LQR tracking term, with $\bar{r}_{\tilde{I}}=-\frac{H-d}{H(d+\delta)}$.

For a dynamics (40), the optimal control problem with cost function (42) corresponds to a classical Linear Quadratic Regulator (LQR) problem with a constant tracking term.

The result, computed and discussed in (Di Giamberardino and Iacoviello, 2018), is a state feedback control law with a reference term. Once the Algebraic Riccati Equation

$$
0=K_{R} \hat{B}_{2} R^{-1} \hat{B}_{2}^{T} K_{R}-K_{R} A_{2}-A_{2}^{T} K_{R}-Q
$$

is solved w.r.t. $K_{R}$, the state feedback optimal control law is given by ((Anderson and Moore, 1989))

$$
\begin{aligned}
\hat{U} & =-R^{-1} \hat{B}_{2}^{T} K_{R} \tilde{X}+R^{-1} \hat{B}_{2}^{T} g_{\bar{r}}= \\
& =K \tilde{X}+r
\end{aligned}
$$

where $g_{\bar{r}}=\left(K_{R} \hat{B}_{2} R^{-1} B_{2}^{T}-A_{2}^{T}\right)^{-1} Q \bar{r}$ and $Q \bar{r}=$ $\left(00 q_{\bar{r}_{\tilde{I}}} 00\right)^{T}, K=-R^{-1} \hat{B}_{2}^{T} K_{R}$ is the gain matrix as in (4) while $r=R^{-1} \hat{B}_{2}^{T} g_{\bar{r}}$ is the constant tracking term $r$.

Stability for the linear controlled system is proven in (Di Giamberardino and Iacoviello, 2018). In the same paper, the problem of the unavailability of a measure of all the state variables has been solved computing a linear state observer under the hypothesis that, once only local solutions are available, due to the request of a state feedback control, then also for the observer a linear approach can be sufficient, whose approximation is well compensated by its simplicity of design and implementation.

Then, once verified the detectability property on $\left(A_{2}, C\right)$ in (40) $(i=2)$, the state estimation $\tilde{z}(t)$ of the state $\tilde{X}$ verifying the asymptotic condition $\lim _{t \rightarrow+\infty}\|\tilde{X}(t)-\tilde{z}(t)\|=0$ can be obtained as the state evolution of the Luenberger like linear observer

$$
\dot{\tilde{z}}(t)=\left(A_{2}-G C\right) \tilde{z}(t)+\hat{B}_{2} \hat{U}(t)+G \tilde{y}(t)
$$


with matrix $G$ chosen in order to have all the eigenvalues of the dynamic matrix $\left(A_{2}-G C\right)$ with negative real part.

The whole control system is a dynamical output feedback control with state observer and feedback from the state estimation.

\subsection{Improved Observer Design}

Following what illustrated in Section 3, consider the control law of the form (44) computed solving the LQR control problem on the basis of the linear approximation of the dynamics in a neighbourhood of $X_{2}^{e}$ is obtained. Under the action of this state feedback, the controlled system assumes the form

$$
\dot{X}=f(X)+\hat{g}(X)(K \tilde{X}+r)=F(X, r)
$$

with its linear approximation asymptotically stable. Its equilibrium point can be denoted by $X_{e}^{c}$ : $F\left(X_{e}^{c}, r\right)=0$ and the linear approximation of (46) in the neighbourhood of $X_{e}^{c}$ can be computed; it is given by

$$
\begin{gathered}
\dot{\bar{X}}=A_{c} \bar{X} \\
\text { where } A_{c}=\left.\frac{\partial F(X, r)}{\partial X}\right|_{X=X_{e}^{c}} \text { and } \bar{X}=X-X_{e}^{c} .
\end{gathered}
$$

A local linear observer for the linear approximating dynamics can be designed in the usual form

$$
\dot{\bar{z}}=\left(A_{c}-G C\right) \bar{z}+G \bar{y}
$$

when dealing directly with the linearised dynamics, or

$$
\dot{z}=\left(A_{c}-G C\right) z+G y-A_{c} X_{e}^{c}
$$

expressed in the original state variables and their estimations.

According to the general discussion in Section 3, once the observer has been designed, the control law (44) should be implemented using the state estimate $\bar{z}$ instead of the real but not measurable state $\bar{X}$ (or $z$ instead of $X$ ). Then the control law (44) has to be rewritten as

$$
\begin{aligned}
u & =K \tilde{X}+r=K\left(X-X_{2}^{e}\right)+r= \\
& =K\left(\bar{X}+X_{e}^{c}-X_{2}^{e}\right)+r= \\
& =K \bar{X}+r+K\left(X_{e}^{c}-X_{2}^{e}\right)
\end{aligned}
$$

so that the dynamics (46) under the employment of the observer (48), assumes the expression

$$
\dot{X}=f(X)+\hat{g}(X)(K \bar{z}+r)+\hat{g}(X) K\left(X_{e}^{c}-X_{2}^{e}\right)
$$

to be considered along with the observer dynamics (48). Some manipulations allow to write the dynamics (51) as

$$
\begin{aligned}
\dot{X} & =f(X)+\hat{g}(X)(K \bar{z}+r)+\hat{g}(X) K\left(X_{e}^{c}-X_{2}^{e}\right)= \\
& =F(X, r)+\hat{g}(X) K\left(\bar{z}-\tilde{X}+X_{e}^{c}-X_{2}^{e}\right)= \\
& =F(X, r)+\hat{g}(X) K(z-X)
\end{aligned}
$$

where $z-X$ can be replaced by $\bar{z}-\bar{X}$ or $\tilde{z}-\tilde{X}$ according to the convenience. It is easy to verify by substitution that the whole dynamics (48)-(52) has the equilibrium point $X=X_{e}^{c}, z=X_{e}^{c}(\bar{X}=\bar{z}=0)$. This means that asymptotically the state of the observer and one of the original system are equal. The fact that the asymptotic error is equal to zero can be proved also computing the error dynamics

$$
\dot{e}=\left(A_{c}-G C\right) \bar{z}+G C \bar{x}-F(X, r)-\hat{g}(X) K(z-x)
$$

and evaluating it in a neighbourhood of $X=X_{e}^{c}$, yielding

$$
\begin{aligned}
\dot{e} & =\left(A_{c}-G C\right) \bar{z}+G C \bar{x}-A_{c} \bar{x}-\hat{B}_{c} K(\bar{z}-\bar{x})= \\
& ==\left(A_{c}-G C+\hat{B}_{c} K\right) e
\end{aligned}
$$

Then, with $\sigma\left(A_{c}-G C+\hat{B}_{c} K\right) \in C^{-}$the error goes asymptotically to zero.

It is confirmed what stated in the previous Section: the observer (48) works properly, without producing undesired changes in the system dynamics and converging asymptotically to the system state, once $G$ is designed to have $\sigma\left(A_{c}-G C-\hat{B}_{c} K\right) \in C^{-}$, provided that $\left(A_{c}-\hat{B}_{c} K, C\right)$ is a detectable pair.

\section{NUMERICAL RESULTS AND DISCUSSION}

In this Section, a numerical analysis is performed to show the effectiveness of the proposed solution and to compare it with the previous more classical one.

The values for the parameters in the dynamics (33) adopted for the numerical computations have been taken, for comparative purposes, from (Di Giamberardino and Iacoviello, 2018; Naresh et al., 2009; Di Giamberardino et al., 2017); they have been firstly used in (Massad, 1989) on the basis of epidemiological research conducted at the San Francisco City Clinic:

$$
\begin{array}{ccc}
d=0.02, & \beta=1.5, \quad \delta=0.4, \quad \varepsilon=0.6 \\
\phi=0.95, & \gamma=0.2 \quad \psi=10^{5}, \quad \alpha=0.5 \\
\mu=1, \quad Z=1000 &
\end{array}
$$

Then, $H=\beta-\delta=1.1>0$ so that the equilibrium point $X_{2}^{e}$ exists and it is locally asymptotically stable. Numerically, $X_{2}^{e}=\left(\begin{array}{lllll}0.91 & 0 & 2.34 & 1.08 & 0.9\end{array}\right)^{T} \cdot 10^{3}$.

The linear approximation in the neighbourhood of this equilibrium point is described by the following 
numerical matrices:

$$
A_{2}=\left(\begin{array}{ccccc}
-0.80 & 0.20 & -0.12 & 0 & 0 \\
0 & -0.22 & 0 & 0 & 0 \\
0.78 & 0 & 0.30 & 0 & 0 \\
0 & 0 & 0.24 & -0.52 & 0 \\
0 & 0 & 0.16 & 0.5 & -1.02
\end{array}\right)
$$

$$
\hat{B}_{2}=\left(\begin{array}{cc}
-0.91 & 0 \\
0.91 & 0 \\
0 & -72 \\
0 & 68.40 \\
0 & 3.60
\end{array}\right) 10^{3}
$$

while $C$ is already expressed in a linear form with respect to $X$ in (37).

As far as the controller is concerned, the control law is computed as the solution of the LQR problem with offset (tracking) term defined in Subsection 4.3 once the controllability property has been checked. It is easy to verify that for $A_{2}, \hat{B}_{2}$ in (56) and (57) it holds.

Performing the computations, the LQR reference term $\bar{r}_{\tilde{I}}$ to be used in (44) assumes the value $\bar{r}_{\tilde{I}}=$ $-2.34 \cdot 10^{3}$.

The weights $q=10^{-4}, r_{1}=1, r_{2}=1000$ are chosen in the cost function; motivations can be found in (Di Giamberardino and Iacoviello, 2018).

The solution $K_{R}$ of the Algebraic Riccati Equation (43) gives

$$
K_{R}=\left(\begin{array}{ccccc}
0.07 & -0.01 & 0.14 & 0 & 0 \\
-0.01 & 0.02 & -0.05 & 0 & 0 \\
0.14 & -0.05 & 4.33 & 0 & 0 \\
0 & 0 & 0 & 0 & 0 \\
0 & 0 & 0 & 0 & 0
\end{array}\right) 10^{-6}
$$

and then $g_{\bar{r}}=\left(\begin{array}{lllll}0.05 & 0 & -1.03 & 0 & 0\end{array}\right)^{T} \cdot 10^{-2}$.

The optimal control (44) so obtained, which should drive the state variable $\tilde{I}$ of the linearised system to the reference value $\bar{r}_{\tilde{I}}$, is of the form (44) with

$$
K=10^{-4}\left(\begin{array}{lllll}
-0.71 & 0.25 & -1.78 & 0 & 0 \\
-0.10 & 0.04 & -3.12 & 0 & 0
\end{array}\right)
$$

and

$$
r=\left(\begin{array}{l}
0.41 \\
0.74
\end{array}\right)
$$

Taking into account the state transformation (41), the linear state feedback control law is computed.

When dealing with the observer design, for comparative purpose firstly the previously available solution is recalled in Subsection 5.1 and then the results of the implementation of the one proposed in this paper are reported and discussed.

\subsection{The Classical Solution}

For this solution, the property of detectability for the pair (56) and (37) must be verified. For the present numerical values it can be verified that it holds.

Then, the design procedures for the state observer can be performed.

Since the solution here adopted is based on the use of a linear state observer, the design procedure requires the computation of matrix $G$ in (45) such that the matrix $\left(A_{2}-G C\right)$ is asymptotically stable.

Discussion about the characteristics of the transient in the observer dynamics are reported in (Di Giamberardino and Iacoviello, 2018) and they bring to the choice of the set of eigenvalues $\Lambda=$ $\{-1.0,-1.1,-1.2,-1.3,-1.4\}$ to be assigned to the matrix $\left(A_{2}-G C\right)$. The corresponding numerical value of $G$ is

$$
G=\left(\begin{array}{lllll}
4.18 & 15.31 & 14.87 & 2.19 & 1.56
\end{array}\right)^{T}
$$

\subsection{The Proposed Solution}

Following the procedure described in Section 3, the observer to be designed has the form (24) rewritten as in (25) and here reported for the present case

$$
\dot{z}=\left(A_{c}-G C\right) z+G C x-A_{c} X_{e}^{c}
$$

where $G$ has to be computed, according to (29), after having verified the detectability property for the pair $\left(A_{c}-B_{c} K, C\right)$, in order to have $\sigma\left(A_{c}-B_{c} K-G C\right) \in$ $C^{-}$.

The first step of the procedure is the computation of the equilibrium point $X_{e}^{c}$ for the controlled system (46) under the hypothesis of a state feedback. For the numerical case here addressed, it is

$$
X_{e}^{c}=10^{4}\left(\begin{array}{lllll}
1.0176 & 3.9822 & 0 & 0 & 0
\end{array}\right)^{T}
$$

For the computation of the matrix $A_{c}-B_{c} K, A_{c}$ has to be computed as the Jacobian of the controlled system evaluated in (63), $B_{c}=g\left(X_{e}^{c}\right)$, while $K$ is the output of the LQR optimal control problem previously solved. Performing all the computations, one gets

$$
A_{c}=\left(\begin{array}{ccccc}
1.09 & 0.21 & 1.04 & 0 & 0 \\
-1.11 & -0.23 & -1.34 & 0 & 0 \\
0 & 0 & -0.34 & 0 & 0 \\
0 & 0 & 0.24 & -0.52 & 0 \\
0 & 0 & 0.16 & 0.50 & -1.02
\end{array}\right)
$$


and, then,

$$
\begin{aligned}
& A_{c}+B_{c} K= \\
& =\left(\begin{array}{ccccc}
1.04 & 0.21 & 0.92 & 0 & 0 \\
-1.06 & -0.23 & -1.22 & 0 & 0 \\
-0.76 & -0.01 & -23.40 & 0 & 0 \\
0.72 & 0.01 & 22.15 & -0.52 & 0 \\
0.04 & 0 & 1.31 & 0.50 & -1.02
\end{array}\right)
\end{aligned}
$$

It can be checked that

$$
\operatorname{rank}\left(\begin{array}{c}
C \\
C\left(A_{c}+B_{c} K\right) \\
C\left(A_{c}+B_{c} K\right)^{2} \\
C\left(A_{c}+B_{c} K\right)^{3} \\
C\left(A_{c}+B_{c} K\right)^{4}
\end{array}\right)=5
$$

and then it is possible to compute $G$ so to verify the convergence condition. With the same choice as in the previous case for the eigenvalues of the error dynamics one has

$$
G=10^{3}\left(\begin{array}{lllll}
-0.22 & 1.20 & 0.03 & -0.02 & 0
\end{array}\right)^{T} \quad(67)
$$

The following Section is devoted to the numerical simulations and the discussion of the results.

\subsection{Simulation Results}

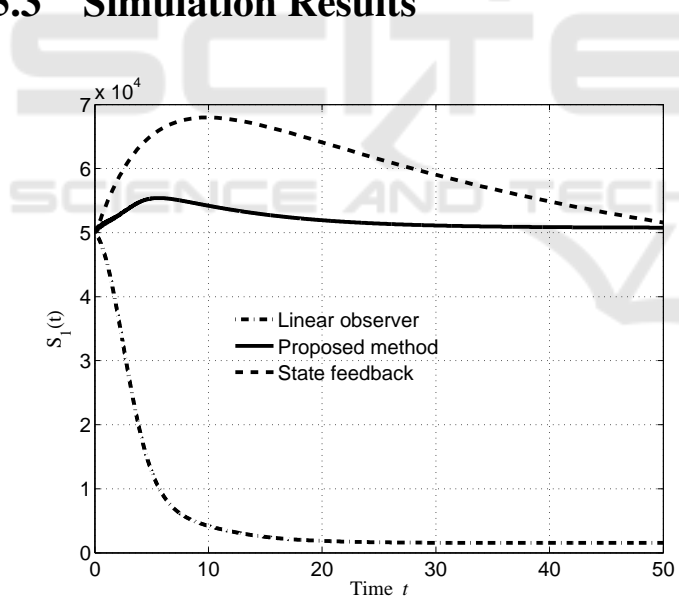

Figure 1: Time history of individuals in $S_{1}(t)$.

Three cases have been simulated to compare their behaviours. One obviously is the application of the procedure proposed in the paper, denoted in the legend of the Figures 1-5 by Proposed method. The numerical values used are the ones reported in the previous Subsection 5.2. The effectiveness of the control scheme can be confirmed by the results depicted in the Figures with the solid lines. In fact, its action produces a fast reduction of the infected $I$, Figure 3, and, consequently, a decrease of the number of the diagnosed patients $P$, Figure 4 , and $A$, Figure 5. At the same time, the number of healthy individuals $S_{1}$

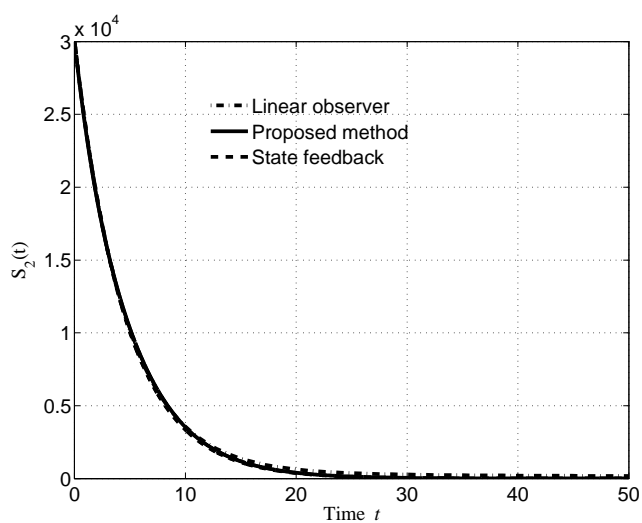

Figure 2: Time history of individuals in $S_{2}(t)$.

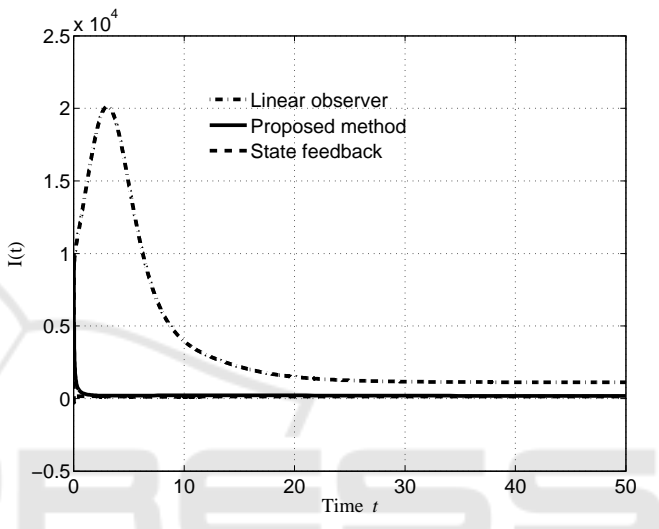

Figure 3: Time history of individuals in $I(t)$.

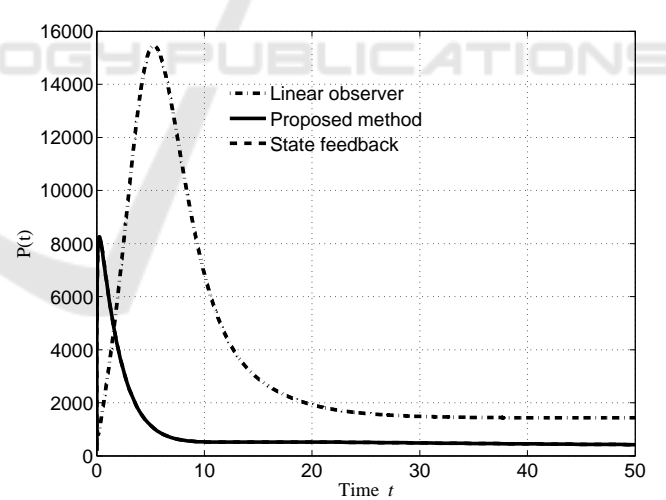

Figure 4: Time history of individuals in $P(t)$.

is maintained sufficiently high, Figure 1, while, due to a reduction of the infection probability, the passage from $S_{1}$ to $S_{2}$ is no more necessary for the spread containment and the individuals in $S_{2}$, reported in Figure 2 , naturally tend to zero by natural death.

Two other cases have been considered. One is the same reported in (Di Giamberardino and Iacoviello, 2018), while the other one is the case in which the state is supposed fully measurable and then the feedback control law can be directly implemented without the necessity of a state observer. 


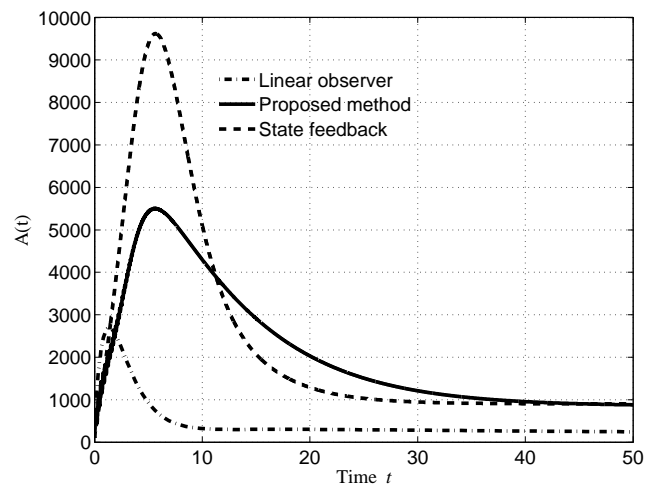

Figure 5: Time history of individuals in $A(t)$.

For each of the five state variables, the results of the simulation for these two cases are reported: the dash-dot line, marked with Linear observer, is devoted to depict the time histories of the state variables in the case of application of the procedure addressed in Subsection 5.1, the one of the previous cited work; the dashed line, for which the denomination in the legend is State feedback, depicts the behaviours of the state variables when the feedback control law is applied using directly a state measurement. These two cases are useful to compare the thee procedures, since in the case of State feedback any negative effect of the observer is avoided, showing the powerful of the control strategy only, while making use of the results for the Linear observer case, it is possible to appreciate the advantages of centring the linear approximation of the observer in correspondence to the new equilibrium point arising from the control action.

\section{CONCLUSIONS}

This paper discusses the problem of the implementation of a state feedback control using an asymptotic state observer. The case in which, for the solution of the control problem, a linear approximation of the dynamics in a neighbourhood of one equilibrium point is the natural framework, also a local linear observer is proposed. The contribution of the paper is in the presentation of the case in which the application of the controller changes the equilibrium point so that, for higher performances, the determination of the linear approximation for the observer design can make use of the knowledge of the new final equilibrium point where the controlled system asymptotically converges. The effectiveness of the proposed solution is also evidenced from the results of numerical simulations, where it is possible to note that in this case the behaviours of the controllers with and without the observer are very similar.

\section{REFERENCES}

Anderson, B. D. O. and Moore, J. B. (1989). Optimal control.

Andrieu, V. and Praly, L. (2006). On the existence of a Kazantis-Kravaris/Luenberger observer. SIAM Journal on Control and Optimization, 45(2):432-456.

Bakare, E. A., Nwagwo, A., and Danso-Addo, E. (2014). Optimal control analyis of an SIR epidemic model with constant recruitment. International Journal of Applied Mathematical Research, 3.

Chang, H. and Astolfi, A. (2009). Control of HIV infection dynamics. IEEE Control Systems Magazine.

Di Giamberardino, P., Compagnucci, L., Giorgi, C. D., and Iacoviello, D. (2017). A new model of the HIV/AIDS infection diffusion and analysis of the intervention effects. $25^{\text {th }}$ IEEE Mediterranean Conference on Control and Automation.

Di Giamberardino, P., Compagnucci, L., Giorgi, C. D., and Iacoviello, D. (2018). Modeling the effects of prevention and early diagnosis on HIV/AIDS infection diffusion. IEEE Transactions on Systems, Man and Cybernetics: Systems.

Di Giamberardino, P. and Iacoviello, D. (2017). Optimal control of SIR epidemic model with state dependent switching cost index. Biomedical Signal Processing and Control, 31 .

Di Giamberardino, P. and Iacoviello, D. (2018). LQ control design for the containment of the HIV/AIDS diffusion. Control Engineering Practice, 77.

Gupar, E. and Quanyan, Z. (2013). Optimal control of influenza epidemic model with virus mutation. European Control Conference.

Iacoviello, D. and Stasio, N. (2013). Optimal control for SIRC epidemic outbreak. Computer Methods and Programs in Biomedicine.

Jun, J. H. (2006). Optimal synthesize control for an SIR epidemic model. $24^{\text {th }}$ IEEE Chinese Control and Decision Conference.

Kazantzis, N. and Kravaris, C. (1997). Nonlinear observer design using Lyapunov's auxiliary theorem. Proc. $36^{\text {th }}$ Conference on Decision \& Control.

Lin, F., Muthuraman, K., and Lawley, M. (2010). Optimal control theory approach to non-pharmaceutical interventions. BNC Infectious Diseases, 10(32):1-13.

Luenberger, D. G. (1964). Observing the state of a linear system. IEEE Trans. on Military Electronics, MIL8(2):74-80.

Massad, E. (1989). A homogeneously mixing population model for the AIDS epidemic. Math. Comput. Modelling.

Naresh, R., Tripathi, A., and Sharma, D. (2009). Modeling and analysis of the spread of AIDS epidemic with immigration of HIV infectives. Mathematical and Computer Modelling, 49.

Pinto, C. and Rocha, D. (2012). A new mathematical model for co-infection of malaria and HIV. 4th IEEE International Conference on Nolinear Science and Complexity. 
Respondek, W., Pogromsky, A., and Nijmeijer, H. (2004). Time scaling for observer design with linearizable error dynamics. Automatica, 40.

Rutherford, A. R., Ramadanovic, B., Michelow, W., Marshall, B. D. L., Small, W., Deering, K., and Vasarhelyi, K. (2016). Control of an HIV epidemic among injection drug users: simulations modeling on complex networks. 2016 Winter Simulations Conference.

Sassano, M. and Astolfi, A. (2019). A local separation principle via dynamic approximate feedback and observer linearization for a class of nonlinear systems. IEEE Trans. Aut. Control, 64(1):111-126.

Sundarapandian, V. (2002). Local observer design for nonlinear systems. Mathematical and Computer Modelling, 35 .

Sundarapandian, V. (2006). Reduced order observer design for nonlinear systems. Applied Mathematics Letters, 19.

Supriatna, A. K., Anggriani, N., Mlanie, and Husniah, H. (2016). The optimal strategy of Wolbachia-infected mosquitoes release program. 2016 Int. Conf. on Instrumentation, Control and Automation (ICA).

Wodarz, D. and Nowak, M. (1999). Specific therapy regimes could lead to long-term immunological control of HIV. Proc. Nat. Acad. Sci., 96(25):1446414469.

Zeitz, M. (1987). The extended Luenberger observer for nonlinear systems. Syst. and Contr. Lett., 9. 